\title{
Anti-FLT3/CD3 BiTE Antibody AMG 427
}

National Cancer Institute

\section{Source}

National Cancer Institute. Anti-FLT3/CD3 BiTE Antibody AMG 427. NCI Thesaurus. Code C156399.

A bispecific T-cell eng ager (BiTE) antibody composed of two single-chain variable fragments (scFv), one directed ag ainst the tumor-associated antigen (TAA) FLT3 tyrosine kinase receptor (Fms-like tyrosine kinase 3; FLT3; FLT-3; CD135; fetal liver kinase-2; FLK2), fused to one that is directed against the CD3 antigen found on Tlymphocytes, with potential immunostimulating and antineoplastic activities. Upon administration of anti-FLT3/CD3 BiTE antibody AMG 427, this bispecific antibody binds to both CD3 on cytotoxic T-lymphocytes (CT LS) and FLT 3 found on FLT3-expressing tumor cells. This activates and redirects CT Ls to FLT3-expressing tumor cells, which results in the CT L-mediated cell death of FLT3-expressing tumor cells. FLT3, a cytokine receptor belong ing to the class III tyrosine kinase receptors, is overexpressed or mutated in most B-lineage and acute myeloid leukemias (AMLs). 